\title{
La ley de justicia y paz y el derecho penal internacional. Algunos aspectos problemáticos*
}

Recibido: febrero 22 de 2008 - Aceptado: junio 25 de 2008

Justice and peace law and international
criminal law. some problematic issues.

Christian Wolffhügel Gutiérrez**

Universidad Sergio Arboleda

\section{Resumen}

Este escrito busca enunciar, con fundamentos teóricos, algunos problemas que la Ley de Justicia y Paz plantea desde dos perspectivas: en primer lugar, la referida al texto legal mismo y, en segundo lugar, la representada por la praxis del proceso de paz. Asimismo, esboza la tensión de tales referentes con algunos apartados del Estatuto de la Corte Penal Internacional, a saber: el preámbulo, el principio de complementariedad y los intereses de la justicia.

\section{Palabras clave}

Proceso de paz, Ley de Justicia y Paz, Estatuto de la Corte Penal Internacional, principio de complementariedad, intereses de la justicia.

\begin{abstract}
This paper attempts to state, based on theoretical foundations, some of the problems presented by the Justice and Peace Law from two perspectives: the first one related to the text itself, and the second to the peace process praxis. By the same token, it outlines the tension of these referents with some sections of the Statute of the International Criminal Court, namely: the preamble, the principle of complementarity, and the interests of justice.
\end{abstract}

\section{key words}

Justice and Peace Law, truth, justice, reparation, International Criminal Court, complementarity, interests of justice.

\footnotetext{
"Artículo de investigación realizado en el proyecto "La Corte Penal Internacional y el proceso de paz colombiano", avalado y financiado por la Universidad Sergio Arboleda y el Grupo de Investigación en Ciencias Penales y Criminológicas "Emiro Sandoval Huertas" Dirección del. Ph.D. Kai Ambos, catedrático de la Universidad de Göttingen.

" " Candidato a Magíster en Derecho de la Universidad Sergio Arboleda. Especialista en Estudios Políticos de la Universidad Sergio Arboleda. Especialista en Instituciones Jurídico Penales de la Universidad Nacional. Correo electrónico: christian.wolffhugel@usa.edu.co
} 


\section{Introducción}

La Corte Penal Internacional es una institución permanente con plena facultad para ejercer, de manera complementaria, su jurisdicción sobre personas nacionales de los estados partes, respecto de los crímenes más graves de trascendencia internacional; en Colombia, algunas de las personas que han perpetrado este tipo de atentados hacen parte de un proceso de paz enmarcado dentro de la Ley de Justicia y Paz.

El presente trabajo pretende esbozar algunas tensiones detectadas entre el articulado del Estatuto de la Corte Penal Internacional y la ya citada Ley 975 de 2005, en especial, los atinentes tanto al preámbulo, al principio de complementariedad y a los intereses de la justicia, como a dos temas muy puntuales: uno, el atingente a la llamada "pena alternativa" prevista en la referida normatividad para aquellos desmovilizados paramilitares que, en desarrollo del proceso de paz adelantado, se han sometido a la Justicia colombiana y, otro, el relativo a los principios de verdad, justicia y reparación los que se examinan de cara a verificar si, en verdad, han o no operado en la práctica.

Al final, se plantean algunas conclusiones para la discusión de tan importante materia en el actual contexto colombiano que, con la mencionada normatividad, le apuesta a la denominada justicia transicional como herramienta llamada a jalonar la paz y, por ende, la lucha contra la justicia privada en nuestro país, de forma muy similar a como ya se ha intentando en otros países, donde el conflicto ha tenido manifestaciones tan profundas como las que hoy impactan a la sociedad colombiana.

\section{De los problemas centrales planteados por la Ley de Justicia y Paz y su Decreto Reglamentario}

\subsection{De los problemas ex ante en el plexo normativo}

Para los fines del presente trabajo se aborda el texto definitivo de la Ley 975, dado que lo concerniente a la genealogía de la misma será objeto de análisis en otro momento.

\section{a. De la pena alternativa}

Un estadio particular del asunto examinado es el atinente a la llamada "pena alternativa" consagrada en la Ley 975; imponible a quienes se han acogido a dicha herramienta, por un lapso que fluctúa entre cinco y ocho años de prisión, a condición de que se cumplan ciertos requisitos y según la gravedad de los delitos cometidos, entre otras exigencias.

Desde esta perspectiva, el primer cuestionamiento que fluye se refiere a si el quantum de la pena alternativa es proporcional a la naturaleza de los delitos, de trascendencia internacional, cometidos por quienes se acojan al proceso de paz adelantado en Colombia.

Al respecto, Uprimny (2006) sostiene que se requiere la existencia de un castigo proporcionado para los delitos de lesa humanidad y para los crímenes atroces; por su parte, Botero y Restrepo (2006) afirman que quienes cometan tales crímenes, deberían ser procesados y condenados a penas también proporcionales. Sostener lo contrario, es decir, preconizar la imposición de penas leves, sería en verdad un mensaje equívoco (Uprimny y Saffón, 2006).

Desde luego, la razón para clamar por la imposición de una pena que resulte proporcional no sólo emerge de la gravedad del comportamiento sino que, para poder honrar a las víctimas, es necesario que se expida una condena equivalente al daño cometido (Robinson, 2003).

Referente a esto, debe recordarse que la Corte Interamericana de Derechos Humanos (en adelante $\mathrm{CIDH})$ en el caso de la "Masacre de la Rochela contra Colombia" al abordar, grosso modo, algunos aspectos de la ley 975 de 2005, dice: en cuanto al referido principio de proporcionalidad de la pena... estima oportuno resaltar que la respuesta que el Estado atribuye a la conducta ilícita del autor de 
la trasgresión debe ser proporcional al bien jurídico afectado y a la culpabilidad con la que actuó el autor, por lo que se debe establecer en función de la diversa naturaleza y gravedad de los hechos. Es más, reiteró, en el párrafo 193, que para que el Estado satisfaga adecuadamente diversos derechos protegidos en la Convención, debe observar el principio de proporcionalidad de la pena.

A su turno, nuestra Corte Constitucional ha sostenido que la pena alternativa, como medida encaminada al logro de la paz, resulta acorde con la Constitución pues no entraña una desproporcionada afectación del valor justicia. Este se preserva mediante la imposición de una pena originaria dentro de los límites establecidos en el Código Penal, proporcional al delito por el cual se condene al agente, y que debe ser cumplida si el desmovilizado sentenciado incumple las obligaciones adquiridas (Sentencia C-370 de 2006). De otra parte, hay quienes sostienen que la disminución sustancial de la pena parece haber vulnerado el interés de la justicia en sentido amplio (Ambos, 2008, p. 167).

Sin duda, la tesis de la Corte Constitucional prodiga un carácter puramente simbólico a la "pena originaria", pues la sanción que en realidad se cumplirá, la "pena alternativa" -salvo que se infrinjan los compromisos--, será de cinco a ocho años de prisión, como ya se dijo. En síntesis, para la Corte Constitucional, resulta claro que la "pena originaria" - a cual puede ser de hasta 60 años de prisión si se tratare de hechos cometidos con posterioridad a la entrada en vigencia de la Ley 890 de 2004, o de cuarenta, tratándose de comportamientos punibles cobijados por la legislación anterior- es proporcional a la gravedad de los crímenes y ella se mantiene incólume al imponer, de manera definitiva, una "pena alternativa" por la realización del mismo hecho, de cinco a ocho años de prisión.

Si bien, la Corte Constitucional encontró ajustada a la Carta Política esa nueva modalidad de la pena, no lo hizo de manera aislada, sino que su razonamiento se hizo depender, directamente, de la efectiva reparación de las víctimas. Por ello, resulta razonable que la reducción de las penas establecida por la norma se encuentre acompañada de la adopción de otras medidas que, como el pago de los daños y la restitución de los bienes, puedan constituir un marco justo y adecuado para alcanzar de forma sostenible la finalidad buscada (Sentencia C-370 del 18 de mayo de 2006). La consecuencia de ello es, entonces, diáfana: si no se repara, imponer la pena alternativa resulta, ineluctablemente, inconstitucional.

\subsection{De los problemas planteados por el Decreto Reglamentario 3391 de 2006}

\section{a. De la revocatoria del beneficio de la pena alternativa}

El artículo 12 del Decreto en cita establece como causal que da lugar a la pérdida del beneficio de la pena alternativa, la situación que se presenta cuando - antes de finalizar el periodo de libertad a prueba- se conozca la existencia de sentencia judicial en la que se establezca la comisión por parte del beneficiario de un delito ocultado por él durante la versión libre, que le sea imputado como miembro del bloque o frente armado organizado al margen de la ley del cual hacía parte y relacionado, directamente, con el accionar del bloque o frente respectivo y su pertenencia al mismo.

Esta causal ha sido objeto de críticas (AmBos, 2008 b), pues en la forma en que está prevista, implica que, además de una sentencia judicial, debe estar rodeada por los siguientes referentes:

a. Que se trate de un delito cometido antes de la desmovilización.

b. Que el delito-ocultado en la versión libretenga relevancia dentro del proceso de paz por su entidad y trascendencia para el esclarecimiento de la verdad. Esta exigencia proviene de manera expresa 
de la sentencia C-370 de 2006 -pues así no fue concebida en la ley 975 de $2005-$ al determinar que " la Corte declarará exequible el inciso $5^{\circ} \mathrm{del}$ artículo 29 en el entendido de que también se revocará el beneficio de alternatividad cuando el beneficiario haya ocultado en la versión libre su participación como miembro del grupo en la comisión de un delito relacionado directamente con su pertenencia al grupo".

c. Que la sentencia se conozca antes de finalizar el periodo de prueba.

En primer lugar, parece claro que el delito no confesado debe tener relevancia dentro del proceso de paz por su entidad y trascendencia, para el esclarecimiento de la verdad. Desde luego, este aspecto resulta ser tan ambiguo que, difícilmente, se podrá revocar el beneficio, pues no se puede precisar cuál es el rasero para determinar si la infracción a la ley penal es o no de importancia. El Decreto no brinda mayores posibilidades hermenéuticas al respecto, por lo que se corre "el peligro de que en la práctica se recurra excesivamente a esta causal para no revocar la pena alternativa una vez otorgada" (Ambos, 2008 b, p.210).

En segundo lugar, la sentencia condenatoria debe conocerse antes de la finalización del período de prueba, esto es, en un lapso que oscilará entre dos y medio y cuatro años, contados con posterioridad al cumplimiento de la pena alternativa. Por supuesto, ubicados en este escenario, resulta bastante problemática la situación que se puede presentar en aquellos eventos en los cuales el "versionado" haya ocultado delitos que sean de trascendencia dentro del proceso de paz, cuya sentencia se conozca después de transcurrido el periodo de prueba, en cuyo caso no habrá lugar a la revocatoria del beneficio.

\subsection{De los problemas "ex post"}

Este escenario -en el que hoy no hay un punto final- merece especial atención, en aras de determinar si principios de la Ley de Justicia y Paz que ex ante fueron avalados por la Corte Constitucional, se mantienen indemnes; o si, por el contrario, han sido embestidos por la realidad del proceso de paz. Se trata, sin duda, de supuestos cardinales cuya óptica dimana de la praxis, por lo que, para los fines de esta reflexión académica, se abordará su contenido teórico a partir de algunos casos en concreto.

\section{a. Del derecho a la justicia}

En relación con este derecho, la Corte Constitucional ha dicho, en la Sentencia C-370 de 2006, que la obligación de investigar las violaciones de derechos humanos forma parte del derecho a la justicia y dicha exigencia -de acuerdo con la jurisprudencia internacional-está aunada a dos elementos adicionales: el plazo razonable y la necesidad de las diligencias orientadas a obtener un resultado satisfactorio.

En ese sentido la CIDH -en los casos de "19 comerciantes contra Colombia", de fecha cuatro de julio de 2005, y el de "Las hermanas Serrano Cruz contra el Salvador", del primero de marzo de 2005, párr. 88 y 66, respectivamente-, sostuvo que "El derecho de acceso a la justicia no se agota en que se tramiten procesos internos, sino que debe además asegurar, en tiempo razonable, el derecho de la víctima o sus familiares a saber la verdad de lo sucedido y a que se sancione a los eventuales responsables".

Por supuesto, de cara a la Ley 975 de 2005, el primer obstáculo que se observa para cumplir con los derroteros ya esbozados radica en las limitaciones del aparato judicial. Este es el caso, pues hasta el momento -con la advertencia de que a la fecha de confección de este escrito se adelanta un proceso de selección de nuevo personal- sólo existen 23 fiscales, 150 investigadores, dos jueces de control de garantías y dos Tribunales de Justicia y paz compuesto por seis Magistrados, que deberán actuar no sólo en torno a los desmovilizados, sino que -aunado a ello- decidirán acerca de las solicitudes de más 
de 70.000 personas que aducen su condición de víctimas (Амвоs, 2008 b) que, con el correr de los días y en la medida en que evolucionan los acontecimientos, se incrementan de manera preocupante hasta llegar a guarismos cercanos a las 130.000. La realidad es que, difícilmente, con estos limitados recursos, se puede pensar de manera seria en cumplir con los presupuestos que reclama el cumplimiento de este piramidal derecho.

\section{b. Del derecho a la verdad}

El artículo $7^{\circ}$ de la Ley 975 de 2005 establece que la sociedad, y en especial las víctimas, tienen el derecho inalienable, pleno y efectivo, a conocer la verdad no sólo sobre los delitos cometidos por los grupos armados organizados al margen de la ley, sino sobre el paradero de las víctimas de los secuestros y las desapariciones forzadas.

A este respecto, la Corte Constitucional, en las tantas veces citada sentencia C-370 de 2006, determinó que el contenido mínimo del derecho de las víctimas a la verdad protege, en primer lugar, el derecho a que los delitos más graves sean investigados. Esto implica que tales comportamientos punibles deben ser investigados y que el Estado es responsable si no hay una indagación seria acorde con la normatividad nacional e internacional.

Por su parte, la CIDH ha sostenido el carácter irrenunciable del derecho de las víctimas o de sus familiares a obtener de los órganos competentes del Estado, el esclarecimiento de los hechos violatorios y de las responsabilidades correspondientes, a través de la investigación y el juzgamiento de los mismos; así las cosas, si se esclarecen las circunstancias en las cuales se llevaron a cabo las gravísimas violaciones de que se trata, se podrá considerar que el Estado ha proporcionado a la víctima y a sus familiares un recurso efectivo de cara a la satisfacción de ese sagrado derecho y ha cumplido con su obligación general de investigar. (Caso Bámaca Velásquez vs. Guatemala Sentencia del 22 de febrero de 2002 párr. 75).
Así mismo, en relación con la dimensión colectiva del derecho a la verdad, se ha señalado que ésta "exige la determinación procesal de la más completa verdad histórica posible, lo cual incluye la determinación judicial de los patrones de actuación conjunta y de todas las personas que de diversas formas participaron en dichas violaciones y sus correspondientes responsabilidades (Caso la masacre de la Rochela vs. Colombia Sentencia del 11 de mayo de 2005).

Por lo anterior, esta prerrogativa no se satisface con el simple procedimiento interno, pues como lo señala Ambos (2008), es necesario que se haga efectivo el derecho de las víctimas y de sus familiares a conocer tanto la verdad de lo sucedido como a que se les imponga a los criminales que causaron el daño la sanción correspondiente.

En relación con este aspecto, debe decirse que, si bien no se puede desconocer la existencia de algunos avances en las investigaciones de cara al esclarecimiento de los casi 14.000 crímenes perpetrados por los grupos paramilitares según estimativos de la Fiscalía General de la Nación, la otra cara de la moneda muestra que la verdad no ha aflorado en los términos y con la dimensión que se esperaba. Este es el caso de Ramón Isaza, quien según su defensa tiene alzheimer, y fue cuestionado por los fiscales debido a los vacíos que tuvo en sus versiones ante Justicia y Paz, no obstante la historia clínica en ningún lado registra el alzheimer: ver el diario El Tiempo, Mayo 3 de 2007. Así mismo, en las cinco sesiones realizadas hasta ahora, 'Báez', importante jefe paramilitar no ha confesado ningún delito diferente a falsedad en documento público y cohecho según lo relatado en El Tiempo, Mayo 31 de 2007.

Por supuesto, no se trata de confesar la comisión de unos delitos sin más, en particular, sino de que la versión libre está llamada a suministrar suficientes herramientas para esclarecer qué sucedió y a quién le ocurrió pues, de no ser así, se conculcarían los irrenunciables derechos de las víctimas; ha habido casos en los 
que si bien se confiesan varios delitos no se dan pistas para saber la verdad de lo ocurrido, ver el diario El Tiempo, Junio 18 de 2007.

\section{c. Del derecho a la reparación}

En relación con este postulado la Corte Constitucional, en la ya citada Sentencia C-370 de 2006, formuló importantes correctivos que fueron retomados en el Decreto Reglamentario 3391 de 2006. Como se recordará, el artículo $8^{\circ}$ de la Ley 975 de 2005 comprende dentro de este derecho las acciones que propendan por la restitución, la indemnización, la rehabilitación, la satisfacción y las garantías de no repetición de las conductas.

Respécto a la restitución cabe citar los casos de la masacre de Mapiripán vs. Colombia, Sentencia del 15 de septiembre de 2004 párrafo 244; las masacres de Ituango vs. Colombia, Sentencia del 1 de julio de 2006 párrafo 347, y Acosta Calderón vs. Ecuador, Sentencia del 24 de junio de 2005 Párrafo 147. En cuanto a la indemnización se ha dicho que, el daño material corresponde a un monto compensatorio que busque reparar las consecuencias patrimoniales de las violaciones; el daño inmaterial puede comprender tanto los sufrimientos y las aflicciones causados a las víctimas, como el menoscabo de valores muy significativos para las personas, así como las alteraciones, de carácter no pecuniario, en las condiciones de existencia de las víctimas mediante dos formas: en primer lugar, el pago de una cantidad de dinero que el Tribunal determine en aplicación razonable del arbitrio judicial y en términos de equidad. En segundo lugar, mediante la realización de actos u obras de alcance o repercusión públicos como es el caso de las masacres de Ituango vs. Colombia Sentencia del 1 de julio de 2006 párrafo 370 y 383 .

En cuanto a la satisfacción, se trata de que estas medidas buscan reparar el daño inmaterial, que no tiene alcance pecuniario, así como también disponer de medidas de alcance o repercusión pública como se dijo en los casos de las masacres de Ituango vs. Colombia Sentencia del 1 de julio de 2006 párrafo 396 y la Masacre de Pueblo Bello sentencia del 31 de enero de 2006 párrafo 264.

Desde el punto de vista teórico, parece que tanto la Ley 975 como el Decreto Reglamentario abordan los principales componentes del derecho a la reparación; sin embargo, en la práctica, no es tan claro el asunto, pues no ha sido posible cumplir con los estándares nacionales e internacionales al respecto. En relación con la entrega de bienes, por ejemplo, se han encontrado diferentes obstáculos, entre los cuales debe recordarse que las sumas de dinero ofrecidas por importantes jefes paramilitares resultan mínimas cuando no exiguas. De 3.257 postulados para justicia y paz, tan sólo 12 han entregado bienes. Entre ellos se cuentan 652 prensas en regular estado, 70 pares de zapatos en regular estado, 4.518 vacas, 19 caballos, 12 predios rurales, 4 lotes urbanos, 2 inmuebles urbanos, 5 vehículos, 859 millones de pesos en efectivo y un televisor de 29 pulgadas en mal estado, según lo expuesto en el diario El Tiempo de Abril 16 de 2008.

\section{Del escenario de la Corte Penal Internacional}

Por medio de la ley 742 de $2002^{1}$ se incorporó al ordenamiento colombiano el Estatuto de Roma que creó la Corte Penal Internacional, a cuyos efectos fue necesario modificar de forma previa el contenido del artículo 93 de la Carta Política, de cara a permitir la inclusión de sus contenidos normativos en el ordenamiento interno y evitar así eventuales contradicciones con lo dispuesto en el texto constitucional (Aponte, 2007).

La estructura del Estatuto, según Ambos (1999), es consecuencia de un difícil proceso con tintes políticos y jurídicos adelantado a lo largo de muchos años, en la búsqueda de soluciones a los diversos problemas que el asunto traía aparejado, por consenso, lográndose refundir los distintos intereses político criminales y los diferentes paradigmas legislativos de los países que intervinieron en dicha discusión, en forma aceptable para todos. 
De cara al proceso de paz reglado por la Ley 975 de 2005 y a la tensión que su expedición comportó en relación con los contenidos del Estatuto de la Corte Penal Internacional, bien vale la pena examinar el asunto bajo tres referentes, a saber: el preámbulo, el principio de complementariedad y los intereses de la justicia.

\section{a. Del Preámbulo}

El Preámbulo del Estatuto de la Corte penal Internacional, en relación con los crímenes más graves de trascendencia para la comunidad internacional, afirma su decisión de poner fin a la impunidad de los autores de dichos latrocinios. Por supuesto, el objetivo delineado es el de asegurar que los crímenes internacionales no queden en la impunidad, como bien lo han expuesto estudiosos de la talla de Young (2002), Robinson (2003) y Moreno (2007). Bajo esa traza, emerge el primer interrogante acerca de si la Ley 975 de 2005 Ley de Justicia y Paz, en su concepción y sobretodo en la práctica, garantiza que el fenómeno de la impunidad no se presente en el caso colombiano.

Ambos (1997), por ejemplo, plantea que el concepto de impunidad abarca dimensiones legales, sociales, culturales y económicas. En esa línea de análisis expone diversas formas de aparición del asunto, así: impunidad legal-material, impunidad procesal y la impunidad como problema estructural, haciendo un análisis de cada una de ellas.

Resulta de especial interés una forma de impunidad -dentro de la procesal- que se presenta "en el caso de que no exista compensación y rehabilitación de la víctima de las graves violaciones de derechos humanos" (Ambos, 1997 p.37). Esta forma de impunidad, que si bien se pretendió evitar con los derroteros decantados por la Corte Constitucional, se presenta en la práctica, a través de diversos casos que generan en este momento serias dudas, acerca de la efectiva posibilidad de reparar a los miles de víctimas (Velásquez, 2007). Desde luego, esta no es una conclusión definitiva en la medida en que el proceso está aún en curso. Es así como en las manifestaciones dadas por José Gregorio Mangones, ex jefe del frente 'William Rivas' de las autodefensas que comandaba 'Jorge 40', dijo que solo cuenta con una camioneta que está a nombre de su compañera sentimental. Las declaraciones las hizo en su tercera sesión de versión libre ante la Fiscalía de Justicia y Paz. Para la reparación se ocupará el comandante general del Bloque Norte ('Jorge 40'), "soy subalterno de él y hará la reparación", ver: en el diario colombiano El Tiempo de Agosto 22 de 2007.

Ahora bien, para otros (Frühling, 2004), habrá impunidad no sólo cuando el autor de un delito escapa a su procesamiento penal, sino también cuando, al ser declarado culpable, es sancionado con penas no proporcionales a la naturaleza y a la gravedad de la conducta punible ejecutada; desde luego, esto implica plantear de la mano del autor citado un test de proporcionalidad entre la naturaleza y gravedad de la conducta punible y la pena. De la mano de lo anterior, en el informe de Diane Orentlicher (2005), experta independiente encargada de actualizar el conjunto de principios para la lucha contra la impunidad, se definió el susodicho fenómeno de la siguiente manera: una infracción de las obligaciones que tienen los Estados de investigar las violaciones, adoptar medidas apropiadas respecto de sus autores, especialmente en la esfera de la justicia, para que las personas sospechosas de responsabilidad penal sean procesadas, juzgadas y condenadas a penas apropiadas, de garantizar a las víctimas recursos eficaces y la reparación de los perjuicios sufridos de garantizar el derecho inalienable a conocer la verdad y de tomar todas las medidas necesarias para evitar la repetición de dichas violaciones.

Esta postura adquiere relevancia directa en relación con la figura de la pena alternativa examinada que, como ya se dijo, debe estar precedida del logro de la verdad, la justicia y la reparación efectivas, pues, si ello no se torna posible, la concesión de tal beneficio no sólo devendría en inconstitucional sino que al tenor 
de lo dicho, abriría de par en par, las puertas para que reinara la impunidad en estos ámbitos.

\section{b. Del principio de complementariedad art. 17 del Estatuto}

Este apartado está destinado a regular la relación entre la Corte Penal Internacional en adelante CPI y las jurisdicciones nacionales; por ello, busca un equilibrio entre el ejercicio de la jurisdicción penal de los Estados y el interés de la comunidad internacional, erigiéndose en la disposición más importante del Estatuto en lo que se refiere a los Estados parte (Ambos, 2008 C). Este principio, según Meyer (2006), es una de las piedras angulares de la CPI.

La lógica de la complementariedad es que la CPI tenga esa oportunidad de ejercer jurisdicción penal, de tal manera que pueda dar un paso más donde las cortes nacionales fracasan al actuar (Burke-Withe, 2005).

Así, pues, la CPI procederá únicamente, según indica Ambos (1999 y 2008 c), si la jurisdicción nacional no está dispuesta o es incapaz de perseguir un delito de su competencia, esto es, si no cumple con sus obligaciones. Por ello, a los estados se les da, según Robinson (2003), la primera oportunidad para procesar.

Ahora bien, cabe preguntar, cómo determinar la falta de voluntad o la incapacidad de persecución penal por parte de un Estado. En relación con la primera cuestión, la falta de voluntad, Ambos (1999) plantea tres hipótesis a saber, en primer lugar, cuando un Estado incorpore un procedimiento sólo aparente, con el propósito de sustraer a la persona interesada de la persecución penal; en segundo lugar, cuando se verifique una dilación procesal incompatible con una intención de persecución penal y, en tercer lugar, cuando el proceso no se sustancie de manera independiente o imparcial.

Frente a la segunda, la incapacidad de persecución, tenemos el escenario propio de un colapso total o sustancial de la jurisdicción nacional, cuando no se consigue hacer comparecer al acusado o no se logra proporcionar los medios de prueba necesarios. $\mathrm{Al}$ respecto, si bien la norma presenta diversas posibilidades, para el caso colombiano se ha dicho que "en algunas de ellas podría ser consecuente la aplicación del Estatuto de Roma. Serán problemas de interpretación, cruzados por toda suerte de presiones o intereses políticos (Aponte, 2007 p. 225).

Ahora bien, el test de complementariedad, de acuerdo con Ambos (2008 c), está determinado por un análisis normativo del artículo $17 \mathrm{del}$ Estatuto, que se mide por la calidad de los procesos y la falta de voluntad e incapacidad del modelo doméstico.

En cuanto al test de la falta de voluntad e incapacidad, el resultado depende de la seriedad del compromiso del gobierno, por un lado, hacia la paz como objetivo supremo del proceso $y$, por el otro, hacia la justicia para las víctimas en cuanto sea posible sin poner seriamente en peligro la paz (Ambos, 2008 c, p. 149)

En relación con el proceso colombiano hay que decir que, amén de los problemas decantados, en la práctica parecen florecer otros que deberán ser objeto de análisis, bajo el criterio normativo del artículo 17 del Estatuto en toda su extensión.

En este momento, existen problemas estructurales. En primer término: la propensión a que sólo se sometan a la ley 975 los mandos paramilitares que tengan sentencias condenatorias en firme, por la principalísima razón de ser más atractiva la "pena alternativa". Por ejemplo cuando se desmovilizó 'Juancho Prada' purgaba una condena de 32 años de cárcel, véase El Tiempo, Febrero 14 de 2007; igualmente Mancuso fue condenado por la masacre de El Aro a 40 años, véase El Tiempo, Diciembre 18 de 2006; Francisco Villalba purga una condena de 33 años y 4 meses de prisión, véase el diario El Tiempo, de abril 23 de 2007. En segundo término, en relación con los mandos medios no 
ha habido mayor preocupación a pesar de ser quienes, en muchos casos, han sido los principales responsables de graves violaciones a los derechos humanos y son los que, además, poseen toda la información acerca de las estructuras militares y financieras de los grupos armados al margen de la ley (Ambos, 2008 b).

\section{c. De los intereses de la justicia, art. 53 del Estatuto}

De acuerdo con este artículo el Fiscal tiene la posibilidad de decidir si inicia o no una investigación teniendo como faro los intereses de la justicia ${ }^{2}$. Se trata, pues, del principio de oportunidad, que además de encontrarse plasmado en el art. 53 del Estatuto, está implícito en la estructura de la etapa de la investigación (Ambos, 2008 b). Según Robinson, (2003), este artículo señala que el Fiscal debe iniciar el trámite a menos que dé las bases razonables para no hacerlo, para ello debe tener en cuenta "el interés de la justicia"; es pues, el criterio del Fiscal el que determina si se investiga o no (Young, 2002).

Por ello, Meyer (2008) sostiene que, una vez se determine la admisibilidad del caso al tenor del art. 17 del Estatuto, el Fiscal deberá considerar, teniendo en cuenta la gravedad del crimen y los intereses de las víctimas, si hay razones sustanciales para creer que una investigación no redundará en interés de la justicia; en tal sentido, es posible la existencia de una situación admisible al tenor del art.17 del Estatuto que, sin embargo, sea excluida en atención al "interés de la justicia" (Ambos, 2008 c).

Ahora bien, anótese que, en relación con el término "intereses de la justicia", no hay consenso entre los estudiosos en torno a los alcances y al significado de esta expresión (Ambos, 2005). Una interpretación a la luz del objeto y propósitos del Estatuto permite inferir que la omisión de la verdad, entre otras, va en contravía del mismo. Según el Derecho internacional una interpretación de los "intereses de la justicia", como mecanismo evasivo para que los autores de los peores crímenes eviten ser procesados debido a un proceso de paz, también resulta contraria a sus derroteros.

La existencia de un proceso de paz no se funda, per se, en un obstáculo para iniciar una investigación por parte del Fiscal de la CPI; la seriedad y satisfacción de los estándares internacionales, al interior de un proceso de aquéllos, es lo que precisa tal posibilidad.

Robinson (2003) en relación con el alcance de la expresión "intereses de la justicia", hace un planteamiento en cuya virtud la CPI enfocará sus baterías en relación con las cúpulas de las organizaciones, pues no es lo mismo para la sociedad el tratamiento que se le dé a quien creó tal situación, que el dispensado a los autores de un bajo perfil; así entonces, la preocupación girará en torno a las cúpulas, a su actividad (Ambos, 2004), pues, según Stahn (2005), se encaminará la persecución hacia un número selecto de victimarios de alto nivel.

Se trata, según Ambos (2008 b), de una limitación ratione personae, debiéndose sancionar a los miembros más importantes. Bajo esa óptica, pues, se satisfarían los intereses de la justicia y, tal vez, no habría base razonable para una investigación, si se castiga a los miembros más significativos de una organización.

Empero, para arribar a esa conclusión en el caso colombiano, sería indispensable, en primer lugar, castigar a los mismos -(que no se sabe si son todos los que están)- a penas adecuadas; en segundo lugar, sería indefectible conocer la verdad de lo sucedido en relación con lo que hicieron; $y$, en tercer lugar, se tendría que reparar a los miles de víctimas. Como se observa, se trata del cumplimiento de estándares que en este momento no se vislumbran de una manera clara.

\section{Conclusiones}

En efecto, en primer lugar, la paz como derecho de talante constitucional es una meta a 
la que el Estado no puede renunciar; sin embargo, para tal cometido se deben proteger valores como el de la justicia bajo la premisa de su observancia ex post, esto es en la práctica.

En segundo lugar, la praxis del proceso de paz ha revelado que para este momento los postulados de la verdad, la justicia y la reparación, como fueron concebidos en la Ley y depurados por la Corte Constitucional, no están cumpliendo a cabalidad su finalidad. No obstante, puede haber un viraje que en la práctica corrija este punto de quiebre.

En tercer lugar, si bien es cierto que la admisibilidad de causas por parte de la CPI es uno de los aspectos más delicados de la aplicación del Estatuto (FLORES, 2005), es posible que un proceso de paz como el colombiano, finalmente, controvierta el principio de complementariedad.

En fin, en cuarto lugar, de lo señalado en esta exposición se infiere que si bien la Ley de Justicia y Paz, es una herramienta para combatir la justicia privada y jalonar el proceso de paz que hoy lidera el Gobierno Nacional, también es cierto que no es descartable la posibilidad de que intervenga la Corte Penal Internacional como tribunal competente para conocer de aquellos atentados calificados como delitos de lesa humanidad. No obstante, el cómo y el cuándo penderá de la evolución de las actuaciones penales hoy en curso en Colombia y de los resultados de las mismas.

\section{Notas}

1 Ley y Protocolo por ella aprobados y declarados exequibles por la Corte Constitucional mediante Sentencia C-578 de 30 de julio de 2002.

2 Human Rights Watch policy paper: The meaning of «the interests of justice» in article 53 of the Rome Statute, 2005. Recuperado 10 de marzo en: http://www.hrw.org/campaigns/icc/ docs/ij070505.pdf

\section{Referencias}

Ambos, K. (1997). Impunidad y Derecho penal internacional, un estudio empírico dogmático sobre Colombia, Bolivia Perú, Chile y Argentina: Diké.

Ambos, K. \& Guerrero, O. (1999). El Estatuto de Roma de la Corte Penal Internacional. Bogotá. Universidad Externado de Colombia.

Ambos, K. (2004a). Problemas seleccionados en torno a los crímenes más graves (core crimes). En el Derecho Penal Internacional. Editorial jurídica Gustavo Ibáñez.

Ambos, K (2004b). Impunidad, Corte Penal Internacional y Colombia. En: Seminario internacional Corte Penal Internacional instrumento de paz para Colombia. Fundación país libre y Konrad - AdenauerStiftung.

Ambos, K. (2008 a). El fundamento jurídico del interés de la justicia, lineamientos sobre exenciones de responsabilidad penal, en particular amnistías. En: E1 marco jurídico de la justicia de transición. Editorial Temis.

Ambos, K. (2008 b). El proceso de paz en Colombia a la luz del Derecho penal internacional, apuntes sobre la Ley 975 de 2005 y el decreto reglamentario 3391 de 2006. En: El marco jurídico de la justicia de transición. Editorial Temis.

Ambos, K. (2008 c). Procesos de paz y CPI. En: El marco jurídico de la justicia de transición. Editorial Temis.

Aponte, A. (2007). El Estatuto de Roma y los limites del derecho penal en escenarios de alta conflictividad: el caso colombiano. En: Dificultades jurídicas y políticas para la ratificación o implementación del 
Estatuto de Roma de la Corte Penal Internacional Contribuciones de América Latina y Alemania. Georg August (Ed.): Universität-Göttingen, Konrad Adenauer Stiftung, Temis.

Botero, C. \& Restrepo, E. (2006). Estándares internacionales y procesos de paz en Colombia. En: ¿Justicia transicional sin transición? Verdad Justicia y reparación para Colombia. Centro de estudios de Derecho, Justicia y Sociedad.

Burke-Withe, W. (2005). Complementarity in Practice: The International Criminal Court as Part of a System of Multi-level Global Governance in the Democratic Republic of Congo. Leiden Journal of International Law 18 , p. $557-590$.

Flores, S. (2005). La admisibilidad de casos ante la Corte Penal Internacional. En: La Corte Penal Internacional una visión iberoamericana: Editorial. Porrúa.

Frühling, Michael (2004). Reflexiones sobre los principios concernientes al Derecho a la Verdad, a la Justicia y a la Reparación. Seminario internacional Corte Penal Internacional instrumento de paz para Colombia. Fundación país libre y Konrad Adenauer- Stiftung.

Human Rights Watch policy paper: The meaning of "the interests of justice" in article 53 of the Rome Statute, 2005. Extraído el 10 de abril de 2007 desde http://www.hrw.org/ campaigns/icc/docs/ij070505.pdf

Meyer, F. (2006). Compementing Complemetarity. International Criminal Law Review 6, 549-583.

Moreno, L. (2007). Transitional justice in ongoing conflicts. The International Juornal of Transitional Justice. Vol 1, 8-9.
Orenlicher, D. (2005). Updated Set of principles for the protection and promotion of human rights through action to combat impunity. Report of the independent expert to update the Set of principles to combat impunity. Extraído el 10 de abril de 2007 desde http://www. derechos.org/nizkor/impu/ principles.html

Robinson, D. (2003). Serving the Interests of Justice: Amnesties, Truth Comissions and the International Criminal Court. EJIL Vol. 14 No 3 481-505.

Stahn, C. (2005). La geometría de la justicia transicional: opciones del diseño institucional. En: Entre el perdón y el paredón. Preguntas y dilemas de la justicia transicional. Universidad de los Andes.

Uprimny, R. (2006). Las enseñanzas del análisis comparado: procesos transicionales, formas de justicia transicional y el caso colombiano. En ¿Justicia transicional sin transición? Verdad Justicia y reparación para Colombia. Centro de estudios de Derecho, Justicia y Sociedad DeJuSticia.

Uprimny, R. \& Saffón, M. (2006). ¿Al fin, ley de justicia y paz? La ley 975 de 2005 tras el fallo de la Corte Constitucional. En: ¿Justicia transicional sin transición? Verdad Justicia y reparación para Colombia. Centro de estudios de Derecho, Justicia y Sociedad De Justicia.

Velásquez V. F. (2007). Foro A los dos años de expedición de la Ley de justicia y paz. Trabajo presentado en el Seminario de rendición de cuentas 24 de julio de 2007. Bogotá

Young, G. K. (2002). Amnesty and Accountability. U.C. Davis Law Review 35 (January): 427482. 


\section{Sentencias Corte interamericana de Derechos Humanos}

Caso Bámaca Velásquez vs. Guatemala Sentencia del 22 de febrero de 2002.

Caso la masacre de la Mapiripán vs. Colombia Sentencia del 15 de septiembre de 2004.

Caso Acosta Calderón vs. Ecuador, Sentencia del 24 de junio de 2005.

Caso la masacre de la Rochela vs. Colombia Sentencia del 11 de mayo de 2005.

Caso las masacres de Ituango vs. Colombia Sentencia del 1 de julio de 2006.
Caso la Masacre de Pueblo Bello sentencia del 31 de enero de 2006.

\section{Corte Constitucional}

C-578 de 2002 M.P. Manuel José Cepeda Espinosa

C- 370 de 2006 M.P. Manuel José Cepeda Espinosa, Jaime Córdoba Triviño, Rodrigo Escobar Gil, Marco Gerardo Monroy Cabra, Alvaro Tafur Galvis y Clara Inés Vargas Hernández. 